\title{
322 MELANOMA-INTRINSIC HYPOXIA-INDUCIBLE FACTOR- $1 \alpha$ RESULTS IN DIMINISHED T CELL ACCUMULATION WITHIN THE TUMOR MICROENVIRONMENT
}

Emily Higgs, Thomas Gajewski, Jonathan Trujillo*. University of Chicago, Chicago, IL, USA

Background The hypoxia-inducible factor (HIF) system, consisting of the transcription factors HIF- $1 \alpha$ and HIF- $2 \alpha$, mediates cellular adaptation to hypoxia, and can promote cancer progression, invasion, and metastasis. HIF pathway activation in the tumor microenvironment has been implicated in cancer immune evasion; however, a direct causal role for tumor cellintrinsic HIF- $1 \alpha$ and HIF-2 $\alpha$ activation in mediating $\mathrm{T}$ cell exclusion and cancer cell resistance to immune checkpoint inhibitor therapy has not been demonstrated.

Methods We performed gene expression analysis of melanoma tumors in the Cancer Genome Atlas (TCGA) data set to determine whether increased HIF-1 $\alpha$ pathway activation correlated with reduced $\mathrm{T}$ cell-based inflammation. The magnitude of HIF-1 $\alpha$ pathway activation across melanoma samples was determined by applying a quantitative scoring system on the expression of a melanocyte-specific hypoxia-induced, HIF- $1 \alpha$ target gene signature consisting of 81 genes. The Pearson correlation test was used to compare the HIF- $1 \alpha$ activation score and our 160-gene T-cell-inflamed gene signature. To determine the impact of cancer cell-intrinsic HIF- $1 \alpha$ or HIF- $2 \alpha$ activation on the endogenous anti-tumor $\mathrm{T}$ cell response, we developed an inducible autochthonous mouse melanoma model driven by BRAFV600E expression and PTEN-deletion, with or without inducible expression of either a stabilized variant of HIF- $1 \alpha$ or HIF- $2 \alpha$. These murine tumor models are being used to determine the impact of cancer cell-intrinsic HIF-1 $\alpha$ or HIF- $2 \alpha$ activation on tumor sensitivity to anti-PD-1/PD-L1 and anti-CTLA-4 treatment.

Results Gene expression analysis of human melanomas in the TCGA demonstrated a statistically significant inverse correlation between the HIF- $1 \alpha$ activation score and T cell-inflammation score. Braf/PTEN murine melanomas with and without stabilized HIF-1 $\alpha$ expression developed with comparable tumor onset and growth kinetics. Multiparameter immunofluorescence staining of melanoma tissue revealed a significant decrease in tumor-infiltrating $\mathrm{T}$ cells within Braf/PTEN melanoma tumors expressing stabilized HIF- $1 \alpha$ compared to control Braf/PTEN melanomas.

Conclusions Our data demonstrate that tumor-cell intrinsic HIF-1 $\alpha$ activation leads to diminished $\mathrm{T}$ cell accumulation within the tumor microenvironment, which has implications for cancer immunotherapy. The mechanism of this effect is being elucidated. These novel murine models will help elucidate the roles of cancer cell-intrinsic HIF- $1 \alpha$ and HIF- $2 \alpha$ activation in modulating the anti-tumor $\mathrm{T}$ cell response, providing mechanistic insight that will inform the evaluation of novel selective HIF inhibitors, which are showing promising antitumor activity in clinical trials in patients with advanced solid tumors.

http://dx.doi.org/10.1136/jitc-2021-SITC2021.322 